\title{
Effects of sample duration and spaced repetition upon delayed matching-to-sample in monkeys (Macaca arctoides and Saimiri sciureus)
}

\author{
HOWARD L. HERZOG, DOUGLAS S. GRANT, and WILLIAM A. ROBERTS \\ University of Western Ontario, London, Ontario, Canada
}

\begin{abstract}
Delayed matching-to-sample was used to study the effects of sample presentation time and spaced repetition upon delayed matching accuracy in one stumptail monkey and three squirrel monkeys. It was found in Experiment 1 that presenting the sample stimulus for $0.5 \mathrm{sec}$ led to lower matching accuracy than was the case with longer presentation times of $2.5,5.0$, and $10.0 \mathrm{sec}$. Experiments 2 and 3 investigated the effects of temporally spacing the presentations of the sample stimulus. It was found that spaced repetition led to a deterioration of performance relative to massed repetition. These results are similar to the findings of experiments with pigeons and are contradictory to severai previous experiments with monkeys or apes which found no effect of presentation time and a facilitative effect of spaced repetition. It is suggested that the use of monkeys inexperienced in short sample duration matching and tested in operant chambers using a limited set of noncomplex stimuli may be responsible for the discrepancies between these results and those of other experiments with primates.
\end{abstract}

The use of delayed-response procedures has become popular recently as a means of testing shortterm memory in animals (D'Amato, 1973; Roberts \& Grant, 1976; Shimp, 1976; Shimp \& Moffitt, 1974). Delayed matching-to-sample experiments carried out in an operant chamber with pigeons have indicated that two fundamental variables influencing short-term memory in the pigeon are sample duration and delay (Roberts, 1972; Roberts \& Grant, 1974). Level of delayed matching accuracy increases in a negatively accelerated manner as length of presentation of the sample increases, and decreases in the same manner as the delay between sample termination and presentation of comparison stimuli is increased. In a variation of the standard procedure, the sample stimulus may be presented once and then repeated prior to the delay, with an interstimulus interval of varying length separating presentations. As the interstimulus interval is lengthened, matching performance has decreased (Roberts, 1972; Roberts \& Grant, 1974). Taken together, this set of observations suggests a simple rule governing delayed matching-to-sample performance in the pigeon: matching accuracy increases as a function of the length of time a bird is exposed to the sample stimulus and decreases as a function of the length of time a bird is left in the

This research was supported by National Research Council of Canada Grants A7894 and A0443. Requests for reprints should be sent to Douglas S. Grant, Department of Psychology, University of Alberta, Edmonton, Alberta, Canada T6G 2 E9. absence of the sample stimulus, regardless of whether the absence of the sample is programmed as a delay or as an interstimulus interval between sample presentations.

The few experiments that have been conducted with nonhuman primates do not lead to such a simple generalization. In an experiment which varied sample duration, D'Amato and Worsham (1972) found that capuchin monkeys matched equally well at presentation times varying between 0.075 and $0.45 \mathrm{sec}$. However, for two reasons this experiment should not lead us to conclude that monkeys show no effect of presentation time on delayed matching. For one, only a narrow range of short sample durations was used; sample durations ranging from 0.5 to $14.0 \mathrm{sec}$ consistently have yielded effects in pigeons. Another important factor in the D'Amato and Worsham study was the use of monkeys highly trained on delayed matching-to-sample. It is possible that naive monkeys would benefit from extended presentations but that highly trained monkeys would have developed encoding and/or rehearsal strategies which would allow them to maintain the memory of a stimulus long beyond its moment of presentation.

With regard to the effects of spaced repetition upon short-term retention in primates, Robbins and Bush (1973) have reported that spaced repetition of trials on two-choice discrimination problems led to better retention than massed repetition in great apes. Medin (1974) found that introducing an interstimulus interval between sample presentations led to better delayed matching than massing sample 
presentations together in pigtailed monkeys. Both of these experiments suggest an effect directly opposite that found in the pigeon, i.e., spacing events apart in time favors their retention. This seemingly paradoxical effect is not unique to these experiments, however, in that spaced repetition improves retention with human subjects (Bjork, 1970; Hintzman, 1974). Thus, it might be argued from these experiments that spaced repetition affects monkeys and apes differently from birds because primates have developed information processing mechanisms similar to those operating in human subjects. It should be noted, however, that the Robbins and Bush and Medin experiments were carried out in the Wisconsin General Test Apparatus. A more appropriate evaluation of the effects of spaced repetition upon monkeys in comparison to pigeons would seem to require that monkeys be tested in an apparatus similar to that used with pigeons (the operant chamber).

In this paper we report three experiments with monkeys naive with respect to delayed matching designed to provide some information about the effects of sample duration and spaced repetition of the sample upon delayed matching in an operant chamber. The first experiment examines the effect of presentation times ranging from values of 0.5 to $10.0 \mathrm{sec}$. The second and third experiments present data on the effects of spaced vs. massed repetition of the sample stimulus on the accuracy of delayed matching.

\section{EXPERIMENT 1}

\section{Method}

Subjects. The subjects of this experiment were one adult female stumptail macaque (Macaca arctoides), Daisy, and three adult male squirrel monkeys (Saimiri sciureus), Curly, Larry, and Moe. They were fed a ration of monkey chow after each day's testing that maintained them at approximately $90 \%$ of their free-feeding weights. Daisy was completely naive to the matching-to-sample procedure at the beginning of training. The squirrel monkeys had been given prior training on simultaneous matching-tosample with colors and patterns but never had been trained on delayed matching-to-sample.

Apparatus. Daisy was tested in a large primate test chamber, $61 \times 60 \mathrm{~cm}$ (floor dimensions) $\times 61 \mathrm{~cm}$ (wall height), and the squirrel monkeys were tested in a small primate test chamber, $30.5 \times 21.5 \mathrm{~cm}$ (floor dimensions) $\times 27 \mathrm{~cm}$ (wall height). Both of these chambers were housed in double-walled, sound-attenuating cubicles. Each chamber contained a panel with a row of three circular keys $(2.6 \mathrm{~cm}$ in diameter) located $46.5 \mathrm{~cm}$ above the floor in the large chamber and $12 \mathrm{~cm}$ above the floor in the small chamber. The keys were spaced $11 \mathrm{~cm}$ apart in the large chamber and $6 \mathrm{~cm}$ apart in the small chamber, measured center to center. The keys required a force of $15 \mathrm{~g}(0.15 \mathrm{~N})$ for operation. Below the keys was placed a centrally located magazine which dispensed $190 \mathrm{-mg}$ Noyes banana and sucrose pellets. Multistimulus projectors mounted behind each key were used to present colored fjelds and black and white patterns on the keys. The stimuli were blue and red fields, a white " $\mathrm{X}$," and a white ring against black backgrounds. The bars of the " $X$ " measured $1.5 \times 0.3 \mathrm{~cm}$; the outer diameter of the ring was $1.5 \mathrm{~cm}$, and the inner diameter was $0.9 \mathrm{~cm}$.

In the large chamber, a recessed $25-\mathrm{W}$ houselight was mounted in the ceiling of the outer cubicle and covered with a piece of frosted glass. In the small chamber, a $0.1-\mathrm{A} / 28-\mathrm{V}$ houselight was mounted on the panel containing the manipulanda, $5 \mathrm{~cm}$ above the upper rim of the center key. The programming of stimuli, delays, interstimulus intervals, and intertrial intervals was controlled by relays, counters, and timers which were activated by a paper-tape reader. Printing counters recorded choices on each trial.

Procedure. Initially, all four monkeys were trained to press the side keys when illuminated with white light for a reinforcer of either one banana pellet or one sucrose pellet (the pellet feeder contained a mixture of banana and sucrose pellets). In the next stage, a press upon a white center key was required to illuminate the side keys; the number of presses required on the center key to illuminate the side keys and the number of presses required on the side keys to obtain a reinforcer were increased gradually to fixed ratios (FR) of FR 10 on the center key and FR 3 on the side keys.

Once animals were satisfactorily completing the FRs required with white keys, simultaneous matching-to-sample training was initiated. On each trial, two stimuli were presented, one as the sample and matching stimulus and the other as the nonmatching stimulus. A typical trial began with presentation of a white center key. The monkey was required to press this key 10 times to produce a sample stimulus on the key; completion of another FR 10 on the sample then presented the side keys simultaneously with the center key, one side key presenting an identical match to the center key and the other a nonmatching color or pattern. If the matching side key was pressed three times first, all of the key lights went off, a reward pellet was delivered, and a 10 -sec intertrial interval elapsed before the white center key appeared to initiate the next trial. The houselight was illuminated during both the trial and the intertrial interval. If an animal first completed an FR 3 on the nonmatching side key, the key lights were darkened, no reward was delivered, and the houselight was extinguished for a 30-sec blackout period. At the end of the blackout, the center key and side keys were reilluminated and the animal was allowed an opportunity for correction. Correction continued until the matching stimulus was chosen. Thus, an incorrect response was penalized by imposing a delay prior to an opportunity for correction. A corrected response was followed by the intertrial interval and the commencement of the next trial.

Each monkey was tested on six different orders of trials, the orders being used in different random sequences across days for different animals. The trial orders were constructed so that each stimulus occurred equally often as a sample and as the incorrect comparison stimulus; also, each stimulus appeared equally often on the left and right side keys as the correct and incorrect choice. Within these restrictions, the different types of trials occurred in random orders.

Daisy was trained for 80 trials per day, while the squirrel monkeys were given 40 trials per day. Initially, Daisy was trained on simultaneous matching-to-sample using red and blue fields and reached a criterion of $85 \%$ accuracy over a block of 5 days within a period of 7 days. She was then trained on yellow and green fields and reached criterion in a period of 11 days. When next trained with a white " $X$ " and a white ring against black backgrounds, she failed to approach criterion over 11 days. In order to facilitate acquisition with patterns, Daisy was trained on daily sequences which presented 40 trials on the blue and red colors and 40 trials on the " $X$ " and ring patterns. Performance with the colors was consistently above $90 \%$ throughout this training, and criterion was reached on the pattern trials after 16 days of training. The squirrel monkeys initially were given 10 days of training on blue and yellow colors and achieved a level of performance only slightly above chance $(50 \%)$. All three monkeys then were transferred to sequences which presented 20 trials using the " $\mathrm{X}$ " and ring pattern stimuli and 20 trials using blue and 
yellow colors. Matching accuracy was consistently high for all three monkeys from the outset with patterns, averaging over $90 \%$, but only Moe showed improvement on the color trials and reached criterion after 12 days of the mixed-trials procedure. Larry and Curly continued to perform at a level only slightly better than chance on the color problems over 15 days of training.

After the completion of simultaneous matching-to-sample training, each monkey began training on 0 -sec delayed matchingto-sample. Daisy was tested on mixed daily sets of trials which contained half blue and red trials and half " $X$ " and ring trials, and the squirrel monkeys were tested on only " $\mathrm{X}$ " and ring pattern trials. Three modifications of procedure were introduced at the beginning of 0 -sec delayed matching training. Although the requirement of 10 presses on the white center key was continued, length of presentation of the sample stimulus was placed under the control of a timer. As another modification in procedure, the sample stimulus was extinguished at the end of its presentation and the side keys were illuminated immediately. Thus, matching had to be done on the basis of a memory of the previously presented sample stimulus. Finally, correction was no longer used and an incorrect choice led to a 2-sec blackout followed by the intertrial interval. For Daisy, the presentation time of the sample stimulus varied between days, the lengths of presentation being $0.5,2.5,5.0$, and $10.0 \mathrm{sec}$. She was tested for 12 days with each presentation time being used on three randomly selected days. The percentages of correct responses at sample exposure durations of $0.5,2.5,5.0$, and $10.0 \mathrm{sec}$ were $85.8,95.8,92.1$, and 95.8 , respectively. Two of the squirrel monkeys, Curly and Moe, were trained on a single presentation time of $5.0 \mathrm{sec}$ for 6 days, by which time they had met the criterion of $85 \%$ correct over a block of 5 days. One subject, Larry, did poorly at the $5.0-\mathrm{sec}$ level and was switched to a 10.0 -sec sample presentation time on Day 13. Larry remained on this procedure until the criterion of $85 \%$ correct over 5 days was reached on Day 25 .

At the completion of 0-sec delayed matching-to-sample training, each animal began Experiment 1. The design of Experiment 1 involved a factorial manipulation of four levels of presentation time, $0.5,2.5,5.0$, and $10.0 \mathrm{sec}$, and four levels of delay, $0,2,5$, and $10 \mathrm{sec}$. The presentation time variable was manipulated between days, and the delay variable was manipulated within days. Daisy was tested for 64 trials each day, with each delay tested for 16 trials. On half of each day's trials, Daisy was tested with blue and red colors as stimuli, and on the other half of the trials with " $X$ " and ring patterns. Each squirrel monkey was tested for 48 trials each day, with each delay being tested for 12 trials. Only the " $X$ " and ring patterns were used for testing the squirrel monkeys.

Each presentation time was tested on 1 day within a block of 4 days, the order of testing the four presentation times varying from animal to animal and block to block. Experiment 1 was carried out for a period of 32 days (eight blocks). Six different orders of trials were used across days, the orders being used in different random sequences for different animals. Within each order, the occurrence of each stimulus as sample and incorrect comparison stimulus, the left-right position of each stimulus, and the length of delay were balanced. One other change in procedure which was introduced in Experiment 1 was an extension of the intertrial interval to $30 \mathrm{sec}$.

\section{Results}

In Figure 1 retention curves are shown which plot percentage of correct (matching) responses against delay, with presentation time as the parameter. Examination of the curves for the squirrel monkeys reveals that Moe and Larry show lower performance at the $0.5-\mathrm{sec}$ presentation time than at the other three, while Curly's curves differ little from one another. All three squirrel monkeys demonstrate decreasing matching accuracy as a function of increasing delay. An analysis of variance performed on the percentage of correct responses for Curly, Larry, and Moe showed a significant effect of presentation time $[F(3 / 6)=4.79, \mathrm{p}<.05]$ and delay $[F(3 / 6)=$ $15.63, \mathrm{p}<.011$. The effect of blocks (Blocks $1-4$ vs. Blocks 5-8) and all interactions failed to reach significance $(p>.05)$. The curves for the stumptail monkey, Daisy, reveal similar effects of the delay and presentation variables. Her accuracy was markedly lower at the $0.5-\mathrm{sec}$ presentation time, and accuracy declined as delay increased. To permit an analysis of variance to be performed, Daisy's data were partitioned on the basis of performance on oddvs. even-numbered trials within each daily session of trials. The analysis showed a significant effect of presentation time $[F(3 / 3)=14.86, p<.05]$ and delay $[F(3 / 3)=400.77, p<.001]$. The only other significant term in the analysis was the Presentation Time by Delay interaction $[F(9 / 9)=3.62, p<.05]$. This interaction reflects the finding that performance at the 0.5 -sec presentation time differs little from that at the other three presentation times at the $0-\mathrm{sec}$ delay but is markedly lower at the other three delays.

These data suggest clear effects of delay and presentation time. Matching accuracy shows the negatively accelerated drop in performance across delays which is typical of delayed matching experiments. The main effect of sample duration is clearly a lower level of accuracy at the $0.5-\mathrm{sec}$ level than at the other three. It could be argued that this latter finding might reflect poor performance during the

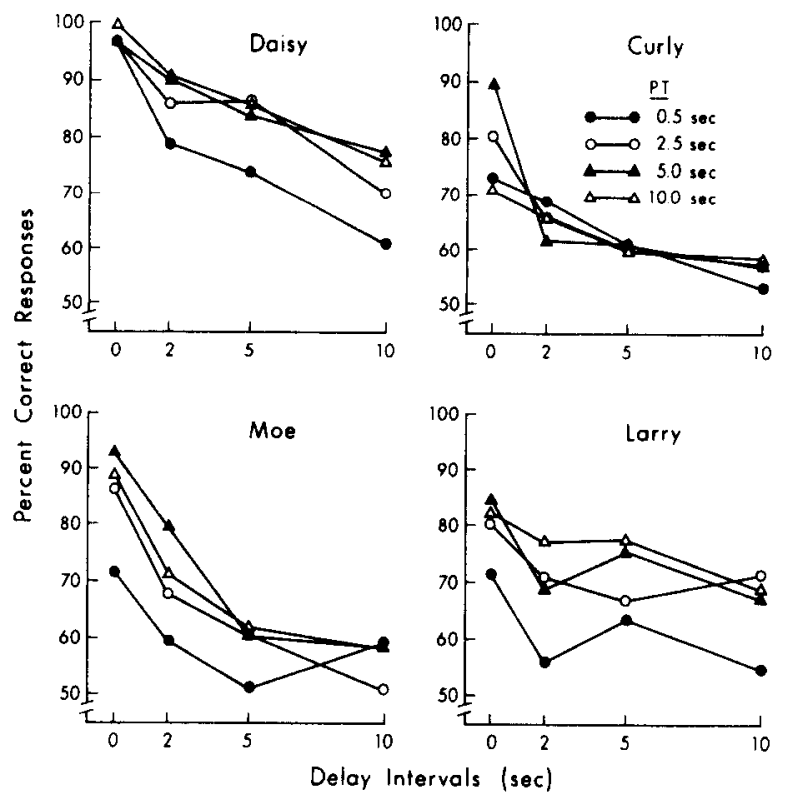

Figure 1. Retention curves plotted for each length of presentation time (PT). Each point on Daisy's curves is based on 128 trials, and each point on the curves of Curly, Moe, and Larry is based on 96 trials. 
early trials of $0.5-\mathrm{sec}$ presentation time sessions, trials which occurred before the monkeys had had an opportunity to adjust to short sample duration. To test this possibility, the first one-quarter of each session of daily trials was excluded from analysis (Trials 1-12 for the squirrel monkeys and Trials 1-16 for Daisy). Collapsing across delay, the squirrel monkeys obtained $62.7 \%$ correct responses at the 0.5 -sec presentation time and $69.5 \%$ correct responses at the other three levels of presentation time combined. Performance was thus 6.8 percentage points lower at the $0.5-\mathrm{sec}$ level when only the last three-quarters of each daily session of trials is considered. A similar analysis of Daisy's data revealed $77.1 \%$ correct at the $0.5-\mathrm{sec}$ level and $85.7 \%$ correct at the other three levels combined, a difference of 8.6 percentage points. For both the squirrel and stumptail monkeys, then, lower accuracy at the 0.5 -sec presentation time was obtained when only the last three-quarters of trials within each session are considered. The effect of the presentation time variable is not, therefore, the exclusive result of preadjustment errors made during the initial trials of short presentation time sessions.

\section{EXPERIMENT 2}

In this experiment, the sample stimulus was presented twice on each trial, the presentations occurring in immediate succession on some trials and spaced $10 \mathrm{sec}$ apart on other trials. The question of interest was whether spaced repetition would hinder delayed matching as it has with pigeons or would facilitate retention as it has in some primate studies, using the Wisconsin General Test Apparatus, and in studies of human memory. One other variable that was studied in this experiment was the degree of chamber illumination present during the interstimulus interval. A surprising result which has emerged from the human memory literature on spacing effects is that a difficult task interpolated between repetitions increases the facilitative effect of spacing on retention (Bjork \& Allen, 1970; Tzeng, 1973). Thus, a condition which produced forgetting of the first presentation aids subsequent retention if a second presentation is given. Since chamber illumination is known to produce forgetting in delayed matching experiments with monkeys (Etkin, 1972; Worsham \& D'Amato, 1973), we tested the possibility that the presence of a bright houselight during the interstimulus interval might lead to a stronger facilitative effect of spacing than would the presence of a dim houselight.

\section{Method}

Subjects. Although the four monkeys used in Experiment 1 were used again in Experiment 2, data are reported for only three. Larry showed obvious signs of ill health throughout the course of Experiment 2, including a worsening of a chronic eye infection, failure to eat his daily ration of food, and listlessness. Larry died within 2 weeks of the termination of the experiment. Because of his ill health and subsequent death, Larry's results, which were atypical, are not reported.

Apparatus. The operant chambers described in Experiment 1 were used in this experiment. Each chamber could be illuminated with either bright or dim houselight. In the large chamber, a $40-\mathrm{W}$ bulb was mounted beside the already present $25-\mathrm{W}$ bulb; illumination of both bulbs consituted the bright houselight condition, whereas illumination of just the $25-\mathrm{W}$ bulb constituted the dim houselight condition. In the small chamber, a rheostat was placed in series with the $0.1-\mathrm{A} / 28 \mathrm{~V}$ houselight; illumination of the houselight with no resistance in the circuit was used as the bright houselight condition, and illumination of the houselight with the rheostat set at $500 \Omega$ was used as the dim houselight condition. The level of illumination under bright and dim houselight conditions in each chamber was measured with a General Electric Type 213 light meter. In each chamber, the illumination was measured directly under the houselight, with the meter placed at the height of the response keys and pointed directly at the houselight. The distance between the meter and the houselight was $5 \mathrm{~cm}$ in the small chamber and $23 \mathrm{~cm}$ in the large chamber. In the small, squirrel-monkey chamber, the bright houselight intensity was $26 \mathrm{fc}$ and the dim houselight intensity was $10 \mathrm{fc}$. In the large, stumptail-monkey chamber, the bright houselight intensity was $80 \mathrm{fc}$ and the dim houselight intensity was $25 \mathrm{fc}$.

Procedure. Three conditions of interstimulus interval (ISI) between sample presentations were used. 0-sec ISI, 10-sec ISIbright houselight, and 10-sec ISI-dim houselight. Four lengths of delay were tested equally often under each of these ISI conditions. With Daisy, the delays used were 5, 15, 30, and $60 \mathrm{sec}$. Since the squirrel monkeys had shown lower levels of performance than Daisy in Experiment 1, they were tested at the shorter delays of $1,2,5$, and $10 \mathrm{sec}$.

On 0-sec ISI trials, a white center key was presented initially; when the monkey completed an FR 10 on the white key, the sample stimulus was presented on the key for $0.5 \mathrm{sec}$. The termination of the sample was followed by the white key again, with an FR 3 requirement preceding another 0.5 -sec presentation of the sample. Requiring three presses on the white center key helped to insure attention to the second presentation. In the bright-houselight and dim-houselight 10 -sec ISI conditions, the initial presentation of the sample was the same as in the 0 -sec ISI condition and was followed by $10 \mathrm{sec}$ of bright or dim illumination of the chamber. At the end of the 10-sec ISI, the white center key was presented for three presses and was followed by a second 0.5 -sec presentation of the sample. Informal observation indicated that monkeys responded to the white center keys with short latencies at both the first and second presentations. Delays began immediately after termination of the second sample presentation. The dim houselight illuminated the chambers during stimulus presentations, delays, and intertrial intervals. During the 10-sec ISI, the houselight was maintained at dim or increased to bright on appropriate trials. A 90 -sec intertrial interval was used throughout the experiment.

The experiment was run for 18 days. Daisy was tested on 64 trials per day, and the squirrel monkeys were tested on 48 trials per day. The stimuli used were the same as in Experiment 1 . There were six different daily orders of trials, and each condition was tested once with each order over the 18 days of testing. The delay variable was manipulated within days such that each delay was tested equally often within a day.

\section{Results}

Retention curves for each monkey are shown in Figure 2. All three monkeys produced similar patterns of generally higher matching accuracy in the 0-sec ISI condition and little difference between the bright and 


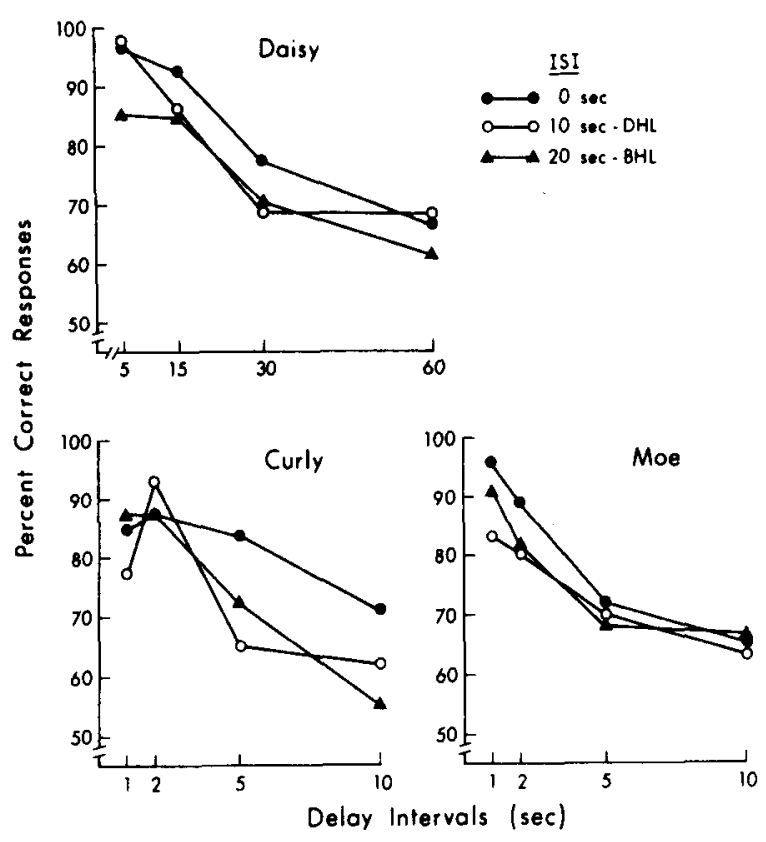

Figure 2. Retention curves plotted for each of the interstimulus interval conditions. Each point on Daisy's curves is based on 96 trials, and each point on the curves of Curly and Moe is based on 72 trials. BHL = bright houselight; DHL $=$ dim houselight.

dim houselight 10-sec ISI conditions. Separate Conditions by Delay by Blocks (Days 1-9 vs. Days 10-18) analyses of variance were performed on the data from the squirrel monkeys and from Daisy, Daisy's data again being partitioned on the basis of performance on odd- vs. even-numbered trials. Although the curves in Figure 2 suggest a deleterious effect of the 10-sec ISI, this effect was not significant in either analysis. The effect of delay was significant in Daisy's analysis $[F(3 / 3)=13.92, p<.05]$ and approached significance in the squirrel monkeys' analysis $[F(3 / 3)=8.66, p<.06]$. The effect of blocks and all interactions were nonsignificant in both analyses.

\section{EXPERIMENT 3}

The result of Experiment 2 suggested that spaced repetitions produce less accurate delayed matching than do massed repetitions. At least two explanations for this finding can be offered, one emphasizing decay of memory and the other emphasizing interference. In both accounts, a spacing interval is held to increase the probability that the memory formed on the basis of the first presentation of the sample will have been lost at the time of the second presentation. Therefore, the proportion of repetition trials which would be functionally equivalent to a singlesample presentation trial would be greater in the spaced condition than in the massed condition. Since Experiment 1 demonstrated higher matching accuracy with increased sample exposure duration (i.e., repetition), massing would be expected to produce a higher level of accuracy than spacing.

The two accounts differ in terms of the property of the spacing interval which is held to be critical for producing the deleterious effect of spacing. According to a decay explanation, it is the passage of time which is critical, since the probability that a memory will be forgotten increases as a function of time. In contrast, according to an interference notion it is not the passage of time per se which is critical but rather the conditions which prevail during the spacing interval. In the case of Experiment 2, it could be held that the presence of illumination from the houselight during the spacing interval caused the memory formed on the basis of the first presentation to be forgotten. Taking the interference notion a step further, it is conceivable that the interference produced by the houselight illumination may have masked a facilitative effect of spacing which might be revealed under conditions of a dark spacing interval. A measure of plausibility is lent to the interference account by delayed matching studies in which the presence of houselight illumination during a delay interval has led to decreased accuracy relative to a dark-delay control in both monkeys (Etkin, 1972; Worsham \& D'Amato, 1973) and pigeons (Grant \& Roberts, 1976).

The decay and interference explanations are clearly not mutually exclusive in that both decay and interference effects may have combined to produce the results of Experiment 2. However, Experiment 3 was performed to determine whether the passage of time per se represents a sufficient condition for obtaining a deleterious effect of spacing by conducting all sessions in the absence of houselight illumination. Moreover, the present experiment provides a test of the possibility that spacing repetitions might increase matching accuracy when the spacing interval is spent in complete darkness.

\section{Method}

Subjects. The squirrel monkeys, Curly and Moe, were the subjects of this experiment. Daisy was not tested because she had been transferred to another experiment, and Larry had died.

Apparatus. The apparatus used in Experiment 3 was exactly the same as that used in Experiment 2.

Procedure. During the approximately 1 month which intervened between Experiments 2 and 3, Curly and Moe continued to be trained on delayed matching. Since matching accuracy increased substantially during this period, the delays were lengthened to $1,10,20$, and $40 \mathrm{sec}$ and a third, longer spacing interval $(20 \mathrm{sec})$ was employed. The three ISIs were 0, 10, and $20 \mathrm{sec}$. Each daily session of trials was conducted with the houselight off at all times and, therefore, the animal spent the ISI in complete darkness. Each ISI was tested equally often with each delay. Each presentation of the sample lasted $0.5 \mathrm{sec}$, with an FR 10 required on the white center key for exposure of the sample on the first presentation and an FR 3 required for the second presentation. Both monkeys were tested using ring and " $x$ " patterns as sample 
and comparison stimuli. The intertrial interval remained at $90 \mathrm{sec}$.

The experiment was carried out over an 18-day period, with ISI conditions tested between days such that each condition was tested once within each 3 -day block. Six different orders of daily trials were used, and each order was paired equally often with each ISI condition. There were 48 trials of testing each day, and each delay was tested on 12 of these trials.

\section{Results}

Curves showing retention at each ISI are presented for Curly and Moe in Figure 3. Examination of these curves indicates that performances was lower in the $20-\mathrm{sec}$ interstimulus interval condition than at the 0 and $10-\mathrm{sec}$ interstimulus interval conditions. This effect is strongly apparent at the 20 - and $40-\mathrm{sec}$ delays for Curly and appears at all four delays in the case of Moe. Performance under the 0 - and 10-sec interstimulus interval conditions differed little. A Conditions by Delay by Blocks (Days 1-9 vs. Days 10-18) analysis of variance revealed significant effects of ISI conditions $[F(2 / 2)=212.99, p<.01]$ and of delay $[F(3 / 3)=9.52, p<.05]$. The only other significant term in the analysis was the Conditions by Delay interaction $[F(6 / 6)=5.49, p<.05]$. This interaction reflects the finding that the difference between the 20-sec ISI condition and the other two conditions increased with increasing delay.

\section{GENERAL DISCUSSION}

Taken together, these experiments provide a pattern of results for the monkeys tested which is similar to previous findings with the pigeon. In Experiment 1 , it was found that accuracy on delayed matching increased from a presentation time of $0.5 \mathrm{sec}$ to longer presentation times of $2.5 \mathrm{sec}$ or more. However, increases beyond $2.5 \mathrm{sec}$ led to only slight increases in accuracy in our monkeys. In contrast, our studies employing the pigeon have demonstrated a pronounced increase in accuracy across a wide range of presentation times (Grant, 1976; Roberts \& Grant, 1974). In Experiments 2 and 3 spaced repetition hindered matching accuracy, a result which is again in line with findings from pigeon experiments. However, a word of caution is again in order. Although quantitative comparison across species and experiments is difficult, such comparison indicates that the deleterious effect of spacing repetitions is more pronounced in the pigeon than in the monkey (see Roberts \& Grant, 1974). Thus, although the present findings with the monkey are similar to previous findings with the pigeon, they are not identical. In general, the variables of presentation time and spaced repetition seem to have more powerful effects in pigeon than in monkey delayed matching.

The present finding that increases in presentation time led to greater delayed matching accuracy in inexperienced monkeys, taken in conjunction with

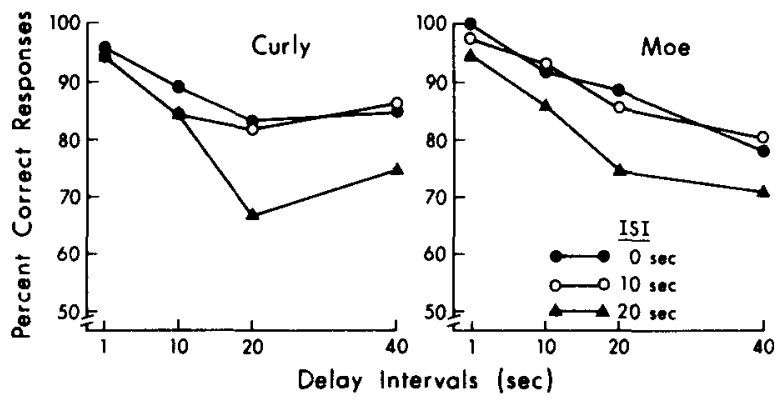

Figure 3. Retention curves plotted for three interstimulus intervals (ISI). Each point on these curves is based on 72 trials.

D'Amato and Worsham's (1972) finding that delayed matching accuracy was independent of presentation time in experienced monkeys, suggests that experienced monkeys may overcome the effects of exposure duration. A recent study by Devine, Jones, Neville, and Sakai (1977), however, suggests that level of delayed matching experience per se is not a critical variable controlling exposure duration effects in the monkey. Employing sample durations of 0.1 , 2.0 , and $4.0 \mathrm{sec}$ with rhesus monkeys, they also found that accuracy in delayed matching was higher at the longer sample durations. Moreover, both experienced and inexperienced animals showed comparable effects. Devine et al. suggested that experience leads to an elimination of presentation time effects only if that experience is with short sample duration matching. The D'Amato and Worsham animals had such short sample duration experience, whereas the experienced animal in the Devine et al. study did not.

Evidence from an experiment by Grant (1976) indicates that experience with short sample duration matching does not eliminate exposure duration effects in the pigeon. Grant found significant increases in delayed matching accuracy across presentation times of $1,4,8$, and $14 \mathrm{sec}$, using birds which had previously received over 16,000 trials of training, of which over 4,000 involved a sample duration of $1 \mathrm{sec}$ or less. These findings suggest the interesting possibility that a common rule governs the performance of pigeons and monkeys naive with respect to short presentation time matching, but that experience with such short presentation time matching results in the development of new techniques for remembering in the monkey but not in the pigeon.

The experiments contained in this report indicate that an ISI occurring between successive repetitions of the sample reduces delayed matching accuracy. In Experiment 2, a 10-sec ISI lowered performance relative to a 0 -sec ISI, although this effect was not significant. In Experiment 3, a 20-sec ISI significantly lowered accuracy relative to 0 - and $10-\mathrm{sec}$ ISIs. The discrepant effects of the 10-sec ISI in Experiments 2 and 3 can be understood readily when the 
substantial improvement in delayed matching which is evident in Experiment 3 is considered. Collapsing across conditions, matching accuracy in the squirrel monkeys dropped from $86.1 \%$ at the 1 -sec delay to $64.1 \%$ at the $10-\mathrm{sec}$ delay in Experiment 2 and from $96.1 \%$ at the $1-\mathrm{sec}$ delay to $79.2 \%$ at the 40 -sec delay in Experiment 3. We attribute this vast improvement in performance to both the beneficial effects of the 1 month of intervening training and to the beneficial effects of conducting all sessions in Experiment 3 in the absence of the houselight, a procedure which is known to improve delayed matching in monkeys (Etkin, 1972; Worsham \& D'Amato, 1973). The higher accuracy in Experiment 3 reflects an increased ability to retain the sample stimulus following its termination, an ability which should be manifest whether the sample is withdrawn prior to a spacing interval or a delay. It is clear that any increased ability to retain the sample stimulus memory would mitigate the deleterious effects of an ISI, requiring longer ISIs in order to lower accuracy.

The deleterious effect of spaced repetitions of the sample stimuli suggested by the present experiments contrasts with other experiments employing primates which report a facilitative effect of a spacing interval. This discrepancy may result from differences in the procedures used. Robbins and Bush (1973), employing pictures as stimuli, tested great apes in a Wisconsin General Test Apparatus on a series of two-choice simultaneous discrimination problems. Each problem was presented for three trials and spacing was manipulated by interpolating various numbers of trials on other problems between successive repetitions of the same problem. Between Repetitions 1 and 2 , either 0,2 , or 10 intervening trials were interpolated, and either 2 or 10 between Repetitions 2 and 3. Medin (1974), also using the Wisconsin General Test Apparatus, tested pigtail monkeys on delayed matching with "junk" stimuli. The sample stimulus was presented three times with either a 6 -sec interval (massed) or a $36-\mathrm{sec}$ interval (spaced) between repetitions. On half of the sessions, only two stimuli were used on all trials, and on the other half of the sessions, a unique set of stimuli was used on each trial. Thus, our procedure differs from that employed in previous spacing research with primates on a number of dimensions, including species of subjects, type of apparatus, complexity of stimuli, number of different stimuli used within a session, and length of ISI.

While any or all of the procedural differences discussed above may be responsible for the discrepant results, Medin's (1974) findings suggest that the number of unique stimuli used with a session may be critical. He found that the facilitative effect of spacing obtained when different stimuli were employed on each trial within a session failed to obtain when only two stimuli were used repeatedly throughout a session. A second procedural difference which may be critical to the discrepancy is suggested by the theory of encoding variability (Madigan, 1969; Melton, 1970). This theory argues that spaced repetition of a stimulus increases the probability that a stimulus will be encoded in different ways at each repetition. Further, the more variable the encoding context of a memory, the more likely is that memory to be retrieved. Since Robbins and Bush employed complex pictures and Medin used three-dimensional junk objects, the complexity of these stimuli may have allowed for differential encoding from one presentation to the next. Using an operant chamber and colored fields or simple patterns as stimuli may not provide sufficient variability for differential encoding to take place on spaced presentations.

There is reason to hypothesize, then, that the number of different stimuli used within a session and/or the complexity of the stimuli may be critical to the discrepancy discussed above. Each hypothesis can be tested readily by repeating our procedures and including the critical procedural addition. Including sessions during which either two stimuli or trialunique stimuli are used would allow an assessment of the importance of the number of stimuli used within a session. Including sessions during which either complex or noncomplex stimuli are used would allow an assessment of the importance of this procedural variable. To the extent that support were found for either hypothesis, it would account nicely for our failure to obtain a facilitative effect of spacing in Experiments 2 and 3. However, the fact that spacing actually hindered delayed matching requires the use of some additional mechanism such as decay of memory.

\section{REFERENCES}

BjoRK, R. A. Repetition and rehearsal mechanisms in models of short-term memory. In D. A. Norman (Ed.), Models of human memory. New York: Academic Press, 1970. Pp. 307-330.

Bjork, R. A., \& AlLEN, T. W. The spacing effect: Consolidation or differential encoding? Journal of Verbal Learning and Verbal Behavior, 1970, 9, 567-572.

D'Amato, M. R. Delayed matching and short-term memory in monkeys. In G. H. Bower (Ed.), The psychology of learning and motivation: Advances in research and theory (Vol. 7). New York: Academic Press, 1973. Pp. 227-269.

D'Amato, M. R., \& Worsham, R. W. Delayed matching in the capuchin monkey with brief sample durations. Learning and Motivation, 1972, 3, 304-312.

Devine, J. V., Jones, L. C., Neville, J. W., \& Sakai, D. J, Sample duration and type of stimuli in delayed matching-to-sample in rhesus monkeys. Animal Learning \& Behavior, 1977, 5, 57-62.

ETKIN, M. W. Light produced interference in a delayed matching task with capuchin monkeys. Learning and Motivation, 1972, 3, 313-324. 
GRANT, D. S. Effect of sample presentation time on long-delay matching in the pigeon. Learning and Motivation, 1976, 7, 580-590.

Grant. D. S., \& Roberts, W. A. Sources of retroactive inhibition in pigeon short-term memory. Journal of Experimental Psychology: Animal Behavior Processes, 1976, 2, 1-16.

Hintzman, D. L. Theoretical implications of the spacing effect. In R. L. Solso (Ed.), Theories of cognitive psychology: The Loyola symposium. Potomac, Md: Erlbaum, 1974. Pp. 77-99.

MADIGAN, S. A. Intraserial repetition and coding processes in free recall. Journal of Verbal Learning and Verbal Behavior, 1969, 8, 828-835.

MEDIN, D. L. The comparative study of memory. Journal of Human Evolution, 1974, 3, 455-463.

Melton, A. W. The situation with respect to the spacing of repetitions and memory. Journal of Verbal Learning and Verbal Behavior, 1970, 9, 596-606.

Roвbins, D., \& Bush, C. T. Memory in great apes. Journal of Experimental Psychology, 1973, 97, 344-348.

RoBERTs, W. A. Short-term memory in the pigeon: Effects of repetition and spacing. Journal of Experimental Psychology, 1972, 94. 74-83.

Roberts, W. A., \& Grant, D. S. Short-term memory in the pi- geon with presentation time precisely controlled. Learning and Motivation, 1974, 5, 393-408.

Roberts, W. A., \& GRant, D. S. Studies of short-term memory in the pigeon using the delayed matching-to-sample procedure. In D. L. Medin, W. A. Roberts, \& R. T. Davis (Eds.), Processes of animal memory. Hillsdale, N.J: Erlbaum, 1976. Pp. 79-112.

Sнimp, C. P. Short-term memory in the pigeon: Relative recency. Journal of the Experimental Analysis of Behavior, 1976, 25, $55-61$.

Shimp, C. P., \& Moffitt, M. Short-term memory in the pigeon: Stimulus-response associations. Joumal of the Experimental Analysis of Behavior, 1974, 22, 507-512.

Tzeng, O. J. L. Stimulus meaningfulness, encoding variability, and the spacing effect. Journal of Experimental Psychology, 1973, 99, 162-166.

Worsham, R. W., \& D'Amato, M. R. Ambient light, white noise, and monkey vocalization as sources of interference in visual short-term memory of monkeys. Journal of Experimental Psychology, 1973, 99, 99-105.

(Received for publication December 2, 1976; revision accepted June $2,1977$. ) 